\title{
Desafios no atendimento à saúde da criança por médicos da Estratégia de Saúde da Família
}

\author{
Challenges in children's health care by physicians in the Family Health Strategy \\ Desafíos en la atención a la salud infantil por médicos de la Estrategia de Salud de la Familia
}

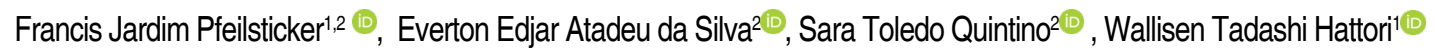 \\ ${ }^{1}$ Universidade Federal de Uberlândia (UFU), Uberlândia, MG \\ ${ }^{2}$ Centro Universitário de Patos de Minas (UNIPAM), Patos de Minas, MG
}

\section{Resumo}

Introdução: $O$ atendimento em Saúde da Criança por médicos da Estratégia de Saúde da Família (ESF) fundamenta-se em recomendações do Ministério da Saúde e da Sociedade Brasileira de Medicina de Família e Comunidade. Objetivo: Identificar as dificuldades dos médicos da ESF para ofertarem assistência em Saúde da Criança no município de Patos de Minas/MG, já que a assistência na Atenção Básica $(A B)$ é prestada pela ESF em quase todo o território neste município. Métodos: Foi aplicado questionário estruturado aos 42 médicos que atuam na ESF, com questões específicas relacionadas ao perfil profissional e da equipe da ESF e quanto às ações em Saúde da Criança na anamnese, exame físico, exames laboratoriais e orientações. Resultados: Responderam ao questionário 32 médicos (76\%). O principal motivo para estarem na ESF foi o interesse pelo trabalho na $\mathrm{AB}(78 \%)$. As dificuldades mais frequentes relacionadas à equipe foram a falta de realização dos grupos de puericultura (41\%) e a ausência da puericultura compartilhada com o enfermeiro (41\%). Apontaram que as habilidades clínicas e a abordagem familiar são fatores que dificultam a realização da consulta de puericultura. As dificuldades apresentadas pelos médicos foram quanto às habilidades no exame físico do recém-nascido, principalmente exame dos olhos (59\%), audição (63\%) e avaliação neurológica (53\%). Dentre os testes oftalmológicos a dificuldade variou de $69 \%$ para acuidade visual até $94 \%$ para o teste de cobertura. Dificuldades relacionadas à estrutura física inadequada e/ou inexistência de equipamento ocorreram para a realização do Teste do Olhinho (34\%) e para a aferição de pressão arterial (69\%). Dificuldades devidas ao desconhecimento de recomendações do Ministério da Saúde ocorreram com o rastreamento de anemia (16\%) e do perfil lipídico (41\%). Quanto às orientações $16 \%$ dos médicos não abordam as curvas de Índice de Massa Corporal (IMC) com as famílias, assim como 19\% não abordam violência na infância. As dificuldades identificadas nos médicos quanto às habilidades clínicas, podem estar relacionadas à qualidade de formação na graduação, assim como à ausência ou ineficácia de educação permanente durante o exercício profissional. Conclusão: A assistência à criança em Patos de Minas apresenta falhas, que estão relacionadas a fragilidades dos médicos, à estrutura física e de equipamentos e às equipes da ESF.

Palavras-chave: Estratégia Saúde da Família; Médicos de Família; Assistentes de Pediatria; Assistência Integral à Saúde.

Como citar: Pfeilsticker FJ, Silva EEA, Quintino ST, Hattori WT. Desafios no atendimento à saúde da criança por médicos da estratégia de saúde da família. Rev Bras Med Fam Comunidade. 2021;16(43):2634. https://doi.org/10.5712/rbmfc16(43)2634

\author{
Autor correspondente: \\ Francis Jardim Pfeilsticker. \\ E-mail: francis@unipam.edu.br \\ Fonte de financiamento: \\ não se aplica. \\ Parecer CEP: \\ CAAE: 88748818.7.0000.5152 \\ Procedência: não encomendado. \\ Avaliação por pares: \\ externa. \\ não encomendado. \\ Recebido em: 13/07/2020. \\ Aprovado em: 11/04/2021.
}




\begin{abstract}
Introduction: Child health care by physicians of the Family Health Strategy (FHS) is based on recommendations of the Ministry of Health and the Brazilian Society of Family and Community Medicine. Objective: It is necessary to identify the difficulties of the physicians of the FHS to assist in Child Health in the municipality of Patos de Minas/MG, since the assistance in Primary Care (PC) is provided by the FHS in almost all the territory of this municipality. Methods: A structured questionnaire was applied to the 42 physicians who work at the FHS, with specific questions related to the professional profile and the FHS team in which it is inserted, and regarding child health care actions in the anamnesis, physical examination, laboratory tests, orientations. Results: Answered the questionnaire 32 physicians (76\%). The main reason to be in the FHS was interest in the work of PC (78\%). The most frequent difficulties were the lack of fulfillment of childcare groups (41\%) and the absence of childcare shared with the nurse $(41 \%)$. Pointed out that clinical skills and family approach are factors that make it difficult to perform childcare appointments. The difficulties presented were related to the skills in the physical examination of the newborn, mainly eye examination (59\%), hearing (63\%) and neurological evaluation (53\%). Among ophthalmologic tests, the difficulty ranged from $69 \%$ for visual acuity to $94 \%$ for the coverage test. Difficulties related to inadequate physical structure and/or lack of equipment occurred for the Eye Test (34\%) and for the blood pressure measurement (69\%). Difficulties due to ignorance of recommendations of the Ministry of Health occurred with anemia screening (16\%) and lipid profile (41\%). Regarding the orientations, $16 \%$ of physicians do not address Body Mass Index (BMI) curves with families, and $19 \%$ do not approach issues related of violence in childhood. The difficulties identified in physicians regarding clinical skills, may be related to the quality of undergraduate training, as well as the absence or ineffectiveness of permanent education during professional practice. Conclusions: Childcare in Patos de Minas presents flaws, which are related to the frailties of physicians, the physical structure and equipment and the teams of Family Health Strategy.
\end{abstract}

Keywords: Family Health Strategy; Family Physicians; Pediatric Assistants; Comprehensive Health Care.

\title{
Resumen
}

Introducción: La atención de la Salud Infantil por parte de los médicos de la Estrategia de Salud Familiar (ESF) se basa en las recomendaciones del Ministerio de Salud y de la Sociedad Brasileña de Medicina de Familia y Comunidad. Objetivo: Se hace necesario identificar las dificultades de los médicos de los ESF para realizar el atendimiento de la Salud Infantil en el municipio de Patos de Minas/MG, ya que la asistencia en la Atención Primaria (AP) es proporcionada por el ESF en casi todo el territorio de este municipio. Métodos: Se aplicó el cuestionario estructurado para los 42 médicos que trabajan en los ESF, con problemas específicos relacionados con el perfil profesional y de los equipos de las EFS y las acciones relacionadas con la anamnesis, examen físico, pruebas de laboratorio y orientaciones. Resultados: 32 médicos respondieron el cuestionario (76\%). La razón principal para estar en el ESF fue el interés en trabajar en la AP (78\%). Las dificultades más frecuentes relacionadas conel equipo fueron la falta de grupos de puericultura (41\%) y la ausencia de puericultura compartido con la enfermera $(41 \%)$. Señalaron que las habilidades clínicas y el enfoque familiar son factores que dificultan la consulta de puericultura. Las dificultades presentadas por los médicos fueron las habilidades en el examen relacionadas con el examen físico del recién nacido, principalmente examen ocular (59\%), audición (63\%) y evaluación neurológica (53\%). Entre las pruebas oftalmológicas, la dificultad varió de 69\% para agudeza visual hasta $94 \%$ para la prueba de cobertura. Dificultades relacionadas con una estructura física inadecuada y/o falta de equipamientos para realizar la prueba de los ojitos (34\%) y medir la presión arterial (69\%). Dificultades por desconocimiento de las recomendaciones del Ministerio de Salud se produjo con la detección de anemia (16\%) y el perfil lipídico (41\%). En cuanto a las recomendaciones, el 16\% de los médicos no abordan las curvas del índice de masa corporal (IMC) en las familias, así como el $19 \%$ no aborda la violencia en la infancia. Las dificultades identificadas en los médicos con respecto a las habilidades clínicas pueden estar relacionadas con la calidad de la formación de pregrado, así como como la ausencia o ineficacia de la educación permanente durante la práctica profesional. Conclusiones: El cuidado infantil en Patos de Minas tiene fallas, que están relacionadas con debilidades de los médicos, estructura física, equipamientos, y equipos del ESF.

Palabras clave: Estrategia de Salud Familiar; Médicos de Familia; Asistentes de Pediatría; Atención Integral de Salud.

\section{INTRODUÇÃO}

Os cuidados primários em saúde são essenciais para a garantia do bem-estar biopsicossocial, conforme foi declarado em Alma-Ata há 42 anos e devem ser inseridos nos locais onde as pessoas estão, constituindo o primeiro contato do sistema de saúde ${ }^{1}$ já no início de suas vidas.

Em 2010 o IBGE (Instituto Brasileiro de Geografia e Estatística) evidenciou, que a população infantil, de 0 a 9 anos de idade tem reduzido, principalmente devido a diminuição da taxa de fecundidade. No entanto, sabe-se que o número de crianças ainda é significativo totalizando $15,1 \%$ da população brasileira ${ }^{2}$. Patos de Minas também vem apresentando esta tendência: no ano 2000 havia $17,3 \%$ da população, de crianças de 0 a 9 anos, e em 2009 havia 14,9\% (22.328) da população, de crianças de 0 a 9 anos (estimativa populacional de 149.856 habitantes em 2016$)^{3}$. 
Em 2015 o Ministério da Saúde (MS) criou a Política Nacional de Atenção Integral à Saúde da Criança (PNAISC), com o principal objetivo de reduzir a mortalidade infantil e promover uma vida digna para garantir um desenvolvimento infantil pleno ${ }^{4}$.

A Política Nacional de Atenção Básica (PNAB) foi revisada em 2017 e ratificou que, a Atenção Básica $(A B)$ deve ser organizada preferencialmente, através da Estratégia de Saúde da Família (ESF), sendo o primeiro contato do usuário com a Rede de Atenção à Saúde ${ }^{5}$. O MS define atribuições necessárias para fundamentar a assistência à criança no Caderno de $A B$ sobre crescimento e desenvolvimento. Juntamente com a PNAB de 2012, cujas atribuições foram ratificadas em 2017, e a Sociedade Brasileira de Medicina de Família e Comunidade, abrangem ações de puericultura, rastreamento de alterações oftálmicas, auditivas e níveis pressóricos, acompanhamento de doenças, além de atribuições comuns aos médicos da $A B^{5,6,7,8}$.

Sabe-se que várias dificuldades vêm ocorrendo desde que surgiu a ESF em 1997. Fatores diversos contribuem para seu enfraquecimento: a desvalorização do médico que atua na $A B$ por seus pares, o número excessivo de consultas, o pouco vínculo com a equipe na qual está inserido e a capacitação ineficaz em Saúde da Família ${ }^{9}$. Como o médico da ESF, é um profissional que presta cuidados às diversas faixas etárias, também acompanha a criança desde a fase neonatal ${ }^{10}$.

Aassistência em Saúde da Criança no Brasil vem apresentando diversas fragilidades nos atributos da $A B$. Trabalho de revisão mostrou que, com a ESF, houve melhora da acessibilidade, porém com ineficácia da integralidade e da resolutividade ${ }^{11}$. Ações que deveriam ser realizadas na puericultura não são feitas ou são realizadas de maneira incompleta, como o registro nas escalas de desenvolvimento e nas curvas de crescimento ${ }^{12}$. A falta de adequada comunicação entre os profissionais e usuários das unidades de $A B$ também foi apontada em estudo em uma microrregião de Minas Gerais ${ }^{13}$.

Como o número de crianças de 0 a 9 anos é significativo no Brasil e em Patos de Minas e não se sabe se as atribuições preconizadas na $A B$ são realizadas na assistência a esta faixa etária neste município, é necessário que se identifiquem as dificuldades e fragilidades relacionadas ao atendimento da criança e assim, propor ações de educação em saúde para os médicos que atuam na ESF, viabilizando uma melhoria da assistência neste ciclo de vida. O objetivo deste estudo foi identificar como está o atendimento em Saúde da Criança (de 0 a 9 anos) pelos médicos da Estratégia de Saúde da Família no município de Patos de Minas apontando as dificuldades para a assistência.

\section{METODOLOGIA}

\section{Caracterização da ESF em Patos de Minas}

Em Patos de Minas a ESF possui 40 equipes com uma cobertura de $95 \%$ a $97 \%$ da população, indicando que a assistência em Saúde da Criança deve estar sendo provida pela ESF em quase todo o território.

\section{Participantes}

Este estudo transversal, quantitativo, descritivo foi desenvolvido no município de Patos de Minas, a $415 \mathrm{Km}$ de Belo Horizonte, na macrorregião Noroeste de Minas Gerais. Foram convidados todos os médicos da ESF do município, totalizando 42 pessoas, sendo que dois destes médicos eram residentes de Medicina de Família e Comunidade. 


\section{Instrumento}

Foi elaborado um questionário ${ }^{14}$ baseado em estudo semelhante abordando médicos da Estratégia de Saúde da Família em relação à saúde da mulher ${ }^{15}$.O questionário era estruturado e continha perguntas sobre o perfil do médico, incluindo sua formação, assim como o perfil da equipe de saúde na qual estava inserido. Para o seguimento sobre Saúde da Criança, que englobava a faixa etária de 0 a 9 anos, as questões continham dados sobre anamnese (abordagem sobre antecedentes pessoais, história familiar, desenvolvimento neuropsicomotor, conferência do calendário vacinal, conferência do Teste da Orelhinha), exame físico (do recém-nascido, por se tratar de um exame físico mais completo e minucioso; testes oftalmológicos na infância, aferição de pressão arterial), solicitação de exames laboratoriais (rastreamento de anemia e do perfil lipídico), orientações (profilaxia de anemia, introdução alimentar no lactente, progressão alimentar do lactente, recomendação de imunobiológicos especiais, prevenção de acidentes, promoção de saúde (lazer, alimentação, estimulação do desenvolvimento neuropsicomotor, atividade física, hábitos de leitura, controle do uso de produtos eletrônicos), interação familiar, preenchimento, interpretação e abordagem das curvas de crescimento) e violência (conhecimento sobre as formas de violência e abordagem com a família) conforme recomendações previstas na $A B^{5,6,8}$. Após as questões estruturadas havia uma questão que permitia ao participante justificar o motivo da não realização da habilidade questionada. Houve também a inserção de perguntas abertas onde foi possível discorrerem sobre as principais dificuldades na realização da puericultura e sobre quais assuntos eram abordados com as famílias durante as consultas.

\section{Procedimentos}

Um questionário piloto foi aplicado aos médicos que atuavam em ESF e que estavam matriculados no Programa de Pós-graduação em Saúde da Família da Universidade Federal de Uberlândia. Todos estes médicos do estudo piloto possuíam títulos de MFC ou já haviam concluído a Residência em MFC ou possuíam Especialização em MFC.

Após este procedimento, o questionário foi enviado por correio eletrônico institucional aos médicos que atuavam na ESF em Patos de Minas, utilizando a ferramenta Formulários do Google Docs, e respondido após aceitarem o Termo de Consentimento Livre e Esclarecido. A coleta de dados ocorreu entre dezembro de 2018 e fevereiro de 2019.

\section{Análise de dados}

Os médicos foram divididos em dois grupos em relação ao tempo de formado, utilizando o ponto de corte em 3 anos para o tempo de formado, já que caso o médico percebesse alguma necessidade de melhoria na atuação profissional, como residência ou especialização, houvesse tempo para esta qualificação ${ }^{15}$. Em relação à sua formação em MFC, os participantes foram agrupados se possuíam alguma formação (residência ou especialização) ou experiência comprovada (título pela SBFMC) em MFC.

Realizou-se a análise descritiva das variáveis categóricas apresentando-se as frequências absolutas e percentuais e para as variáveis métricas utilizando-se médias e desvios padrões. Nestas análises, incluiu-se as variáveis que caracterizam os participantes, a equipe da ESF e as principais dificuldades evidenciadas na consulta médica. 


\section{Aspectos éticos}

O estudo foi aprovado pelo Comitê de Ética em Pesquisa da Universidade Federal de Uberlândia (UFU) (Parecer 2.731.737). Não houve participação de pacientes/comunidade no planejamento e condução da pesquisa. Optamos pelo não compartilhamento do banco de dados publicamente dada a dificuldade de proteger o anonimado dos participantes, visto que as características pessoais, de formação e atuação permitem a sua identificação, mas poderão ser disponibilizados a outros pesquisadores mediante apresentação de projeto de pesquisa com aprovação em Comitê de Ética em Pesquisa em Seres Humanos, garantindo o anonimato dos participantes.

\section{RESULTADOS E DISCUSSÃO}

Pôde-se identificar dificuldades médicas na Estratégia de Saúde da Família que, podem prejudicar o cumprimento dos itens preconizados na assistência à Saúde da Criança. Os resultados evidenciaram que, os médicos da ESF de Patos de Minas possuem dificuldades relacionadas tanto com a equipe na qual estão inseridos como quanto a habilidades clínicas, habilidades de comunicação, condições físicas e de insumos para a execução da assistência.

Os questionários foram respondidos por 32 (76\%) dos 42 médicos que trabalham na ESF de Patos de Minas.

A Tabela 1 mostra as características gerais dos médicos e de sua equipe. A média de idade foi de 38,25 anos (DP 9,88 anos), sendo 21 (66\%) profissionais do sexo feminino e 11 (34\%) do sexo masculino.

O principal motivo para atuar na ESF foi o fato de gostar de trabalhar na AB (78\%). Em comparação com outros dois estudos o percentual foi bem mais elevado: Vasconcelos e Zaniboni $(2011)^{9}$ encontraram $25,0 \%$ e Melo et al. $(2014)^{15}$ encontraram 38,4\%.

Observou-se que $84 \%$ dos participantes seguiam a periodicidade recomendada para as consultas de puericultura. Observou-se que todos os participantes que possuíam a formação em MFC seguiam esta periodicidade de consultas. A formação não é essencial, mas talvez seja um facilitador para desenvolver esta ação.

Quanto ao perfil da equipe foi observado que $41 \%$ não realizam o Grupo de puericultura e $41 \%$ não realizam a Puericultura compartilhada entre médico e enfermeiro. Identificou-se que, 28\% das equipes não realizam ambas as atividades. Os grupos operativos são um recurso importante e efetivo para alcançar resultados exitosos em prevenção, promoção e educação em saúde ${ }^{16}$, além de fortalecer o vínculo dos usuários com a equipe de saúde ${ }^{17}$. A Agenda de Compromissos para a Saúde da Criança e Redução da Mortalidade Infantil (2004) recomenda a criação destes grupos para o combate à desnutrição ${ }^{18}$, o que poderia ser extrapolado para diversos outros temas em Saúde da Criança, como a obesidade já que, esta condição vem interferindo no crescimento saudável. Um estudo amplo realizado com a participação da Organização Mundial de Saúde evidenciou que crianças e adolescentes (5 a 19 anos) com obesidade serão mais frequentes no mundo em 2022 do que desnutridos moderados e graves ${ }^{19}$. Quanto à consulta compartilhada, é sabido que a consulta de enfermagem, desde o início da vida da criança é muito importante, pois aborda questões relacionadas à prevenção e promoção de saúde, além de possibilitar a percepção de problemas de saúde precocemente. Este acompanhamento permite se criar um vínculo da equipe de saúde com a criança, principalmente se tal ligação tiver ocorrido durante a gestação ${ }^{20}$. É importante destacar que as equipes que não realizam as duas atividades perdem grandes oportunidades para educação em saúde. 
Tabela 1. Distribuição dos participantes segundo as características do perfil do médico e da equipe da ESF em Patos de Minas - MG, 2018 e 2019

\begin{tabular}{|c|c|c|}
\hline Características & $\mathrm{n}=32$ & $\mathbf{n} \%$ \\
\hline \multicolumn{3}{|l|}{ Perfil do médico } \\
\hline \multicolumn{3}{|l|}{ Tempo de formado } \\
\hline Menos de 3 anos & 7 & 22 \\
\hline Maior ou igual a 3 anos & 25 & 78 \\
\hline \multicolumn{3}{|l|}{ Tipo de faculdade } \\
\hline Pública & 8 & 25 \\
\hline Privada & 21 & 66 \\
\hline Fundações & 3 & 9 \\
\hline Residência ou Especialização em MFC & 11 & 34 \\
\hline \multicolumn{3}{|l|}{ Motivos para trabalhar na ESF } \\
\hline Gosta de trabalhar na $A B$ & 25 & 78 \\
\hline Salário/Emprego/Horário de trabalho & 14 & 44 \\
\hline Gosta do contato com a Comunidade & 18 & 56 \\
\hline Sem outra opção de trabalho & 1 & 3 \\
\hline Aprendizado & 2 & 6 \\
\hline \multicolumn{3}{|l|}{ Tempo de atuação na ESF } \\
\hline Menos de 3 anos & 11 & 34 \\
\hline Maior ou igual a 3 anos & 21 & 66 \\
\hline Perfil da equipe da ESF & 30 & 94 \\
\hline \multicolumn{3}{|l|}{ Equipe como contato preferencial } \\
\hline Programa Saúde na Escola & 29 & 91 \\
\hline Cuidado compartilhado com Pediatra & 27 & 84 \\
\hline Fluxo de encaminhamento adequado (ESF/ Pediatria) & 31 & 97 \\
\hline Acompanhamento do pré-natal (todas ou maioria) & 26 & 81 \\
\hline Grupo de Puericultura (com e sem o NASF) & 19 & 59 \\
\hline Puericultura compartilhada (médico e enfermeiro) & 19 & 59 \\
\hline Periodicidade de Puericultura recomendada pelo MS & 27 & 84 \\
\hline Manejo de problemas de saúde & 31 & 97 \\
\hline Acompanhamento de doenças agudas & 29 & 91 \\
\hline
\end{tabular}

A Tabela 2 mostra as dificuldades quanto à consulta de puericultura, assim como quanto à assistência em relação à anamnese e exame físico. Quanto às dificuldades para a realização da puericultura foram apontados fatores relacionados à família, às habilidades médicas e à equipe da ESF. Quanto à família, a dificuldade relatada foi quanto à falta de adesão às consultas, com altas taxas de absenteísmo. Em relação à habilidade médica, as dificuldades foram relacionadas à falta de educação permanente e falta de especialistas de referência. Relacionados à equipe foram ressaltados a falta de busca ativa e número excessivo de consultas.

A dificuldade mais frequente apontada pelos médicos em relação à consulta de puericultura foi a pouca habilidade clínica para o exame da criança (44\%). Podemos pensar que, talvez a formação médica na graduação e a educação permanente durante o exercício profissional, estejam aquém das reais necessidades dos médicos da ESF. Estudo de um curso de especialização em Saúde de Família realizado com médicos que atuavam na área, evidenciou, após aplicação de pré-teste sobre Saúde da Criança, que os alunos não atingiram $60 \%$ em alguns temas importantes, incluindo acompanhamento do crescimento e desenvolvimento ${ }^{21}$. Esta pesquisa abrangeu médicos com tempos de profissão variados (23,0\% com $<5$ anos; $34 \%$ com 5 a 15 anos; $43 \%$ com > 15 anos), mostrando que tanto a formação na graduação quanto a ineficácia de educação permanente podem estar relacionadas às falhas de habilidades. 
Tabela 2. Distribuição dos participantes segundo dificuldades para a assistência à Saúde da Criança em Patos de Minas - MG, 2018 e 2019

\begin{tabular}{lcc}
\hline Dificuldades dos médicos na consulta & $\mathbf{n}=\mathbf{3 2}$ & $\%$ \\
\hline Dificuldades na Puericultura relacionados à & & 34 \\
Família & 11 & 44 \\
Habilidade médica & 14 & 6 \\
Equipe & 2 & 3 \\
Dificuldades no exame físico do recém-nascido & & 19 \\
Face & 1 & 9 \\
Pele & 6 & 59 \\
Crânio & 3 & 63 \\
Olhos & 19 & 22 \\
Audição (avaliação clínica) & 20 & 16 \\
Orelhas & 7 & 16 \\
Nariz & 5 & 13 \\
Boca & 5 & 6 \\
Pescoço & 4 & 6 \\
Tórax & 2 & 16 \\
Abdômen & 2 & 25 \\
Genitália & 5 & 34 \\
Ânus & 8 & 31 \\
Sistema osteoarticular & 11 & 53 \\
Coluna vertebral & 10 & \\
Avaliação neurológica & 17 & 69 \\
Dificuldades no Teste oftalmológico na infância & & 72 \\
Acuidade visual & 22 & 94 \\
Teste do olhinho & 23 & 91 \\
Teste de cobertura & 30 & 91 \\
Rastreio de estrabismo & 29 & \\
Dificuldades na aferição de pressão arterial & 29 & \\
\hline & & \\
\hline
\end{tabular}

A abordagem familiar foi apontada como a segunda causa de dificuldade (34\%) para a realização das consultas, sendo que a falta de compromisso e adesão das famílias às consultas foram as maiores causas citadas. Estas mesmas causas foram encontradas por profissionais de saúde de unidades básicas em estudo qualitativo, que avaliou a consulta de puericultura sob o olhar dos profissionais e das mães em Belo Horizonte. Estas mães reconhecem que os problemas relacionados ao baixo comparecimento à UBS ocorrem devido à distância da unidade, escassez de recursos financeiros e ao esquecimento da data da consulta. Além disto, as mães não reconhecem o termo puericultura, não dando a real importância ao acompanhamento da maneira que deveria ocorrer ${ }^{22}$. Vitolo et al. (2010) identificaram que os fatores associados ao não acompanhamento da criança foram a insatisfação da família com o serviço, a impossibilidade de levar a criança devido ao emprego dos familiares e o julgamento da não necessidade do acompanhamento ${ }^{23}$, este último fator reforçando o desconhecimento da população sobre o motivo e a importância da puericultura.

No exame físico do recém-nascido, exceto pela avaliação do estado geral, todos os outros itens apresentaram relato de dificuldades ou não realização, devido à falta de habilidade clínica ou algum empecilho (falta de tempo, local físico inapropriado ou ausência de material). É recomendado que se faça o exame completo na primeira consulta da criança ${ }^{6}$. Os de maior dificuldade foram: exame dos olhos (59\%), exame clínico da audição (63\%), avaliação neurológica (53\%).

Houve identificação de dificuldades nos 4 testes oftalmológicos pesquisados, sendo que no Teste do Olhinho se relacionaram à falta de equipamento e/ou ambiente apropriado (34\%). Estes testes são triagem de possíveis alterações que, sendo detectadas, devem ser encaminhadas ao oftalmologista. Os testes necessitam 
ser realizados com periodicidade e com técnica adequada para que realmente seja efetivo como rastreio ${ }^{6}$. A dificuldade com o Teste do Olhinho foi apontada por $72 \%$ dos médicos e é o único teste oftálmico dentre os investigados, que necessita de aparelho e de ambiente físico apropriado, não dependendo somente das habilidades clínicas para sua realização. Os outros testes, apesar de não requerem nenhuma tecnologia para sua realização, apresentaram dificuldade similar ou bem superior à do Teste do Olhinho, chegando a 94\% no Teste de Cobertura, mostrando novamente que uma possível deficiência de formação na graduação e de ausência ou ineficiência de educação permanente pode justificar esta dificuldade.

Em aferição da pressão arterial foi apontada dificuldade por $91 \%$ dos participantes; deste percentual $69 \%$ relataram que não realizam a aferição por falta de manguitos adequados para o tamanho da criança e $19 \%$ relataram não conhecer a recomendação do MS. Estas dificuldades podem comprometer a detecção de alterações passíveis de intervenção. É importante salientar, que a aferição da pressão arterial na infância também é competência do enfermeiro e o Caderno de AB recomenda que seja realizada aos 3 e 6 anos de idade .

No questionamento de solicitação de exames, foi perguntado sobre o rastreamento de anemia e investigação do perfil lipídico. O rastreamento de anemia não foi realizado por $16 \%$ que desconhecem a recomendação, assim como o perfil lipídico não foi solicitado por $41 \%$ dos médicos pelo mesmo motivo. Este desconhecimento pode estar relacionado à falta de formação e de educação permanente em Saúde da Criança, já que foi apontado que não sabem sobre a recomendação do MS.

A Tabela 3 traz alguns aspectos sobre as abordagens e orientações feitas ao final da consulta. Em relação às curvas de crescimento os médicos relatam ser os maiores responsáveis pelo preenchimento (91\%). O preenchimento da curva de IMC é atribuído a outro membro da equipe por $19 \%$ dos participantes. Apenas um médico $(3 \%)$ relatou apresentar dificuldade de interpretação das curvas de peso, estatura e perímetro cefálico e 2 (6\%) relataram dificuldade de interpretação do IMC.

Quanto às orientações fornecidas para as famílias $16 \%$ não abordam sobre o IMC. Observou-se que uma minoria (13\%) possui dificuldade para o preenchimento do IMC, porém, um total de $44 \%$ dos médicos não preenche a curva, sendo que esta função é atribuída a outro membro da equipe apenas por $19 \%$ dos médicos. Portanto, $12 \%$ dos participantes não preenchem a curva de IMC e não possuem na equipe, um profissional que faça este preenchimento. A curva do IMC é uma ferramenta valiosa para a detecção de desvios de crescimento ${ }^{6}$. Deixar de preencher a curva pode ocasionar a falta de abordagem e ausência de orientações quanto a alterações.

Os imunobiológicos especiais, não são recomendados devido a desconhecimento das indicações por $38 \%$ dos médicos, apontando mais uma vez, para uma possível falha na formação e na educação permanente.

Para uma primeira investigação sobre a violência na infância, onde foi perguntado sobre quais assuntos eram abordados nas consultas, foi identificado que o assunto sobre violência não apareceu entre os 32 médicos participantes. Sobre o conhecimento das diversas formas de violência pôde-se evidenciar que, conhecem grande parte: automutilação (88\%), suicídio (81\%), violência intrafamiliar (100\%), violência extrafamiliar (100\%), violência sexual intrafamiliar (100\%), violência sexual extrafamiliar (100\%), violência psicológica intrafamiliar (100\%), violência psicológica extrafamiliar (97\%), bullying (100\%), cyberbullying (94\%), negligência (94\%), privação (91\%) e abandono (97\%). As formas de violência menos conhecidas são as violências coletiva econômica (59\%), coletiva política (66\%), coletiva social ( $81 \%)$, sendo que essa se iguala ao conhecimento quanto ao suicídio. No que se refere a abordagem sobre violência, evidenciou- 
se que 5 médicos (16\%) sempre abordam, outros 21 médicos (66\%) fazem a abordagem às vezes e 6 participantes (19\%) não abordam este assunto, por principalmente não saberem como fazer. As Diretrizes Curriculares Nacionais do Curso de Medicina preconizam que o médico deve possuir habilidades de comunicação que sejam efetivas na relação médico paciente ${ }^{24}$.

Estas mesmas DCN enfatizam que o Curso de Medicina deve se apoiar nas metodologias ativas de aprendizagem, proporcionando ao graduando em medicina o desenvolvimento da capacidade de aprender baseada em sua prática, comprometendo-se com seu processo de formação, sabendo identificar suas necessidades de aprendizagem ${ }^{24}$ e assim, poder perpetuar a busca pelo conhecimento por sua vida profissional. Não foi questionado qual o modelo curricular presente durante a graduação dos participantes para que pudessem ser comparadas as variáveis com o fato de terem estudado ou não em faculdades que adotam metodologias ativas de aprendizagem.

Percebe-se que, muitas dificuldades apontadas pelos médicos participantes, poderiam ser amenizadas ou resolvidas se houvesse um programa de educação permanente, que se desenvolvesse a partir das

Tabela 3. Distribuição dos participantes segundo às ações e dificuldades ao fim da consulta na ESF em Patos de Minas/ MG, 2018 e 2019

\begin{tabular}{lcc}
\hline Ações e dificuldades & $\mathrm{n}=32$ & $\%$ \\
\hline Curva de crescimento & & 44 \\
Não preenche a curva de IMC & 14 & 13 \\
$\quad$ Possui dificuldade para o preenchimento do IMC & 4 & 38 \\
Recomendação de imunobiológicos especiais & 12 & 6 \\
$\quad$ Não, pois desconhece recomendação & 2 & \\
Não, devido à esquecimento & & \\
\hline
\end{tabular}

demandas dos próprios profissionais. O Programa de Educação Permanente (PEP) foi implantado no município de Patos de Minas a partir da Secretaria de Estado da Saúde de Minas Gerais em 2010 e tinha a prerrogativa de aprendizagem baseada na realidade profissional dos médicos. O programa foi abolido em 2015. Não houve estudo no município de Patos de Minas para avaliar o quão benéfico tenha sido sua ação no período. Trabalho descrito por Silvério (2008), que avaliou a percepção dos médicos participantes do PEP em outros municípios, verificou que $95 \%$ apontaram a metodologia com um potencial para mudar a prática; $88 \%$ disseram que tinham interesse pela busca de novos conhecimentos após a metodologia e 90\% tinham interesse em continuar participando do PEP ${ }^{25}$.

Portugal possui um sistema de AB semelhante ao Brasil, com a Unidade de Saúde Familiar (USF) sendo a porta de entrada do sistema de saúde. O médico que integra a equipe é especialista em medicina geral e familiar e é reconhecido entre os outros médicos dos diferentes níveis de assistência, facilita, portanto, a coordenação do cuidado e a assistência eficaz. Lá os processos formativos de educação em saúde são permanentes com discussão de casos, construção de protocolos, atualizações da prática clínica. Estas atividades ocorrem com o apoio de outros especialistas, o que torna a USF mais resolutiva garantindo assim a integralidade da assistência ${ }^{26}$. Ao contrário de Portugal, em Patos de Minas, grande parte dos médicos que atuam na ESF não possuem formação específica em Medicina de Família e Comunidade, o que poderia estar relacionado a uma assistência não integral na Atenção Básica. Esta situação, aliada a falta ou ineficiência de educação permanente, talvez perpetue uma assistência com diversas fragilidades. 
Por se tratar de um estudo com foco na compreensão de aspectos relacionados à ESF e à Saúde da Criança, optou-se por uma amostra de conveniência, o que traz consigo limitações para as generalizações dos achados. Entretanto, esta proposta permitiu a detecção de pontos de maior fragilidade de assistência em Saúde da Criança em um município e pode ser aplicada em outros municípios para identificação das dificuldades na realidade de outros municípios. Em consonância, sabe-se que a Educação Permanente deve ser realizada tendo como foco as demandas dos próprios trabalhadores para que a prática reflexiva consiga implementar melhorias na assistência ${ }^{27}$. Este diagnóstico das dificuldades relatadas é capaz de fornecer os temas a serem abordados com a equipe da ESF, especialmente através da Educação Permanente, para promover melhorias no cuidado.

\section{CONCLUSÕES}

As falhas na assistência em Saúde da Criança na ESF ocorrem não somente devido a fragilidades das habilidades médicas, mas também pela falta de uma abordagem multiprofissional e por uma estrutura ineficaz, incapaz de propiciar a assistência conforme a preconização do Ministério da Saúde e da Sociedade Brasileira de Medicina de Família e Comunidade. As dificuldades evidenciadas pelos médicos podem estar relacionadas à qualidade de formação na graduação, que talvez não enfatize todas as competências necessárias para a adequada assistência em Saúde da Criança na $A B$, assim como à ausência ou ineficácia de Educação Permanente durante o exercício profissional.

\section{Conflitos de interesse}

Os autores declaração não haver.

\section{Contribuições}

Francis Jardim Pfeilsticker: Concepção, aquisição de dados, análise de dados, interpretação de dados, redação preliminar, revisão crítica.

Wallisen Tadashi Hattori: Concepção, delineamento do estudo, análise de dados, interpretação de dados, revisão crítica.

Everton Edjar Atadeu da Silva: aquisição de dados, análise de dados, interpretação de dados, revisão crítica.

Sara Toledo Quintino: aquisição de dados, análise de dados, interpretação de dados, revisão crítica.

\section{Agradecimentos}

Ao Prof. Wallisen Tadashi Hattori pela paciência, dedicação e orientação.

À FIOCRUZ, instituição que coordena o Mestrado Profissional em Saúde da Família (PROFSAUDE), por ter me proporcionado participar de um mestrado, que trouxe mais significado à minha profissão.

\section{REFERÊNCIAS}

1. Organização Mundial de Saúde. Relatório da Conferência Internacional sobre Cuidados de Saúde Primários (Internet). Declaração de Alma-Ata: 1978. [online] [acesso em 2017]. Disponível em: https://www.opas.org.br/declaracao-de-alma-ata 
2. Instituto Brasileiro de Geografia e Estatística [homepage na internet]. Atlas do Censo Demográfico de 2010 [acesso em 2017]. Disponível em: http://censo2010.ibge.gov.br/apps/atlas

3. Secretaria Municipal de Saúde. Plano Municipal de Saúde - Gestão 2014-2017. 2014. Patos de Minas, MG, 2014.

4. Ministério da Saúde (Brasil). Portaria no 1.130, de 5 de agosto de 2015. Institui a Política Nacional de Atenção Integral à Saúde da Criança (PNAISC) no âmbito do Sistema Único de Saúde (SUS) [portaria na internet]. [acesso em 2017]. Disponível em: http://bvsms.saude.gov. br/bvs/saudelegis/gm/2015/prt1130_05_08_2015.html

5. Ministério da Saúde (Brasil). Portaria no 2.436, de 21 de setembro de 2017 [portaria na internet]. Aprova a Política Nacional de AB, estabelecendo a revisão de diretrizes para a organização da AB, no âmbito do Sistema Único de Saúde (SUS). Brasília: Ministério da Saúde; 2017. [acesso em 2017]. Disponível em: http://pesquisa.in.gov.br/imprensa/jsp/visualiza/index.jsp?jornal=1\&pagina=68\&da $\mathrm{ta}=22 / 09 / 2017$

6. Departamento de Atenção Básica. Secretaria de Atenção à Saúde. Ministério da Saúde (Brasil). Cadernos de Atenção Básica 33: Saúde da criança: crescimento e desenvolvimento [internet]. Brasília, DF: Ministério da Saúde, 2012 [acesso em 2017]. Disponível em: http:// bvsms.saude.gov.br/bvs/publicacoes/saude_crianca_crescimento_desenvolvimento.pdf

7. Departamento de Atenção Básica, Secretaria de Atenção à Saúde, Ministério da Saúde (Brasil). Política Nacional de Atenção Básica (Série E. Legislação em Saúde) [internet]. Brasília: Ministério da Saúde; 2012 [acesso em 2017]. Disponível em: http://189.28.128.100/ dab/docs/publicacoes/geral/pnab.pdf

8. Lermen Júnior N. Currículo Baseado em Competências para Medicina de Família e Comunidade. Sociedade Brasileira de Medicina de Família e Comunidade (SBMFC) [Internet]. 2015. [acesso em 2017]. Disponível em: https://www.sbmfc.org.br/noticias/sbmfc-divulgacurriculo-baseado-em-competencias/

9. Vasconcelos FGA, Zaniboni MRG. Dificuldades do trabalho médico no PSF. Ciênc Saúde Coletiva [periódicos na Internet].2011 [acesso em 2018]; 16(Suppl 1): 1497-1504. DOI: https://doi.org/10.1590/S1413- 81232011000700085

10. Giovanella, L, Mendonça MHM. Atenção Primária à Saúde. In: Giovanella L, Escorel S, Lobato LVC, Noronha JC, Carvalho Al. Políticas e sistemas de saúde no Brasil. 2. ed. Rio de Janeiro: Fiocruz; 2012. P. 493-535.

11. Damasceno SS, Nóbrega VM, Coutinho SED, Reichert APS, Toso BRGO, Collet N. Saúde da criança no Brasil: orientação da rede básica à Atenção Primária à Saúde. Ciênc Saúde Coletiva [periódicos na Internet]. 2016 [acesso em 2019]; 21 (9): 2961-2973. DOI: https://doi. org/10.1590/1413-81232015219.25002015

12. Costa GD, Cotta RMM, Reis JR, Ferreira MLSM, Reis RS, Franceschini SCC. Avaliação da atenção à saúde da criança no contexto da Saúde da Família no município de Teixeiras, Minas Gerais (MG, Brasil). Ciênc Saúde Coletiva [periódicos na Internet]. 2011 [acesso em 2019]; 16(7): 3229-3240. DOI: https://doi.org/10.1590/S1413-81232011000800022

13. Silva SA, Fracolli LA. Silva SA, Fracolli LA. Avaliação da assistência à criança na Estratégia de Saúde da Família. Rev. Bras. Enferm. [periódicos na Internet]. 2016 [acesso em 2019]; 69(1): 54-61. DOI: https://doi. org/10.1590/0034-7167.2016690107i

14. Pfeilsticker FJ, Silva EEA, Quintino S, HattoriWT. Questionário para a pesquisa: Desafios no atendimento à Saúde da Criança por médicos da Estratégia de Saúde da Família. Repositório Zenodo. 2020 Jul. Disponível em: https://zenodo.org/record/3941786\#.X_R0HhZ7nlU

15. Melo VH, Rio SMP, Bonito RF, Lodi CTC, Fonseca MTM, Amaral E. Dificuldades dos médicos que atuam na Estratégia Saúde da Família de Minas Gerais para proverem atenção à saúde das mulheres. Rev Bras Med Fam Comunidade [periódicos na Internet]. 2014 [acesso em 2017];9(30):3-12. DOI: https://doi.org/10.5712/rbmfc9(30)550

16. Menezes KKP, Avelino PR. Grupos operativos na Atenção Primária à Saúde como prática de discussão e educação: uma revisão. Cad. saúde colet. [periódicos na Internet].2016 Mar [acesso em 2019];24(1): 124-130. DOI: https://doi.org/10.1590/1414-462X201600010162

17. Branco CKCG, Barbosa ASB, Santos JMCG, Queiroga VE, Cavalcanti FRR. Puericultura em Grupo: uma nova Perspectiva na Atenção à Saúde da Criança: Relato de Experiência. Rev Bras Ciênc Saúde [periódicos na Internet]. 2014 [acesso em 2019]; 18(1):63-68. http:// dx.doi.org/10.4034/rbcs.2014.18.s1.11.

18. Departamento de Ações Programáticas Estratégicas, Secretaria de Atenção à Saúde, Ministério da Saúde (Brasil). Agenda de compromissos para a saúde integral da criança e redução da mortalidade infantil [internet]. 2004a [acesso em 2019]. Disponível em: http://bvsms.saude.gov.br/bvs/ publicacoes/agenda_compro_crianca.pdf.

19. NCD-RisC. Worldwide trends in body-mass index, underweight, overweight, and obesity from 1975 to 2016: a pooled analysis of 2416 population-based measurement studies in 128.9 million children, adolescents, and adults. LANCET [internet]. 2017 [acesso em 2019]; 16;390(10113):2627-2642. https://doi.org/(...)0140-6736(17)32129-3 PMID: 29029897 
20. Souza RS, Ferrari RAP, Santos TFM, Tacla MTGM. Atenção à saúde da criança: práticas de enfermeiros da saúde da família. Rev REME [periódicos na Internet]. 2013 [acesso em 2019]; 17(2):331-339. DOI: https://doi.org/10.5935/1415-2762.20130025

21. Alves CRL, Alvim CG, Junqueira HS, Almeida JSCB, Goulart LMH, Magalhães MEN, et al. Avaliação do conhecimento de alunos do curso de especialização em saúde da família da UFMG sobre a saúde da criança e do adolescente - 2002/2003. Rev Med Minas Gerais [periódicos na Internet]. 2005 [acesso em 2019]; 1(1):213-219. Disponível em: http://rmmg.org/artigo/detalhes/1382

22. Dias PRM. A consulta de puericultura na perspectiva de mães e profissionais de unidades básicas de saúde de Belo Horizonte [internet]. Belo Horizonte. Dissertação [Saúde e Enfermagem] - Universidade Federal de Minas Gerais; 2017 [acesso em 2019]. Disponível em: https://repositorio.ufmg.br/bitstream/1843/ANDO-AQNPZA/1/poliana_reginele_de_melo_dias.pdf

23. Vitolo MR, Gama CM, Campagnolo PDB. Frequência de utilização do serviço público de puericultura e fatores associados. J. Pediatr. (Rio J.) [periódicos na Internet]. 2010 Feb [acesso em 2019]; 86(1): 80-84. DOI: https://doi.org/10.1590/S0021-75572010000100014

24. Ministério da Educação (Brasil). Resolução CNE/CES no 3, de 20 de junho de 2014. Institui Diretrizes Curriculares Nacionais do Curso de Graduação em Medicina e dá outras providências [internet]. Diário Oficial da União, Brasília, DF, 2014 [acesso em 2017]. Disponível em:http://www.fmb.unesp.br/Home/Graduacao/resolucao-dcn-2014.pdf. PMID: 30017874

25. Silvério JB. Programa de educação permanente para médicos de família. Rev Med Minas Gerais [periódicos na Internet]. 2008 [acesso em 2019]; 18(Supl 4): S60-S66. Disponível em: http:// rmmg.medicina.ufmg.br/index.php/rmmg/article/viewArticle/104

26. Santos AM, Giovanella L, Botelho H, Sousa JC. Desafios Organizacionais para Fortalecimento da Atenção Primária à Saúde em Portugal. Rev. bras. educ. med. [periódicos na Internet]. 2015 [acesso em 2019]; 39(3): 359-369. DOI: https://doi.org/10.1590/1981$52712015 v 39 n 3 e 01542014$

27. Ferreira L, Barbosa JSA, Esposti CDD, Cruz MM. Educação Permanente em Saúde na atenção primária: uma revisão integrativa da literatura. Saúde debate [Internet]. 2019 Mar [acesso em 2021]; 43(120): 223-239. DOI: https://doi.org/10.1590/0103-1104201912017 